Oprya Ye. V., Melnyk E. V., Biesieda Ya. V., Kozishkurt Ye. V. Social adaptive potential of mentally ill patients with concomitant pathology. Journal of Education, Health and Sport. 2021;11(07):310-315. eISSN 2391-8306. DOI http://dx.doi.org/10.12775/JEHS.2021.11.07.029

https://apcz.umk.pl/JEHS/article/view/JEHS.2021.11.07.029

https://zenodo.org/record/5660810

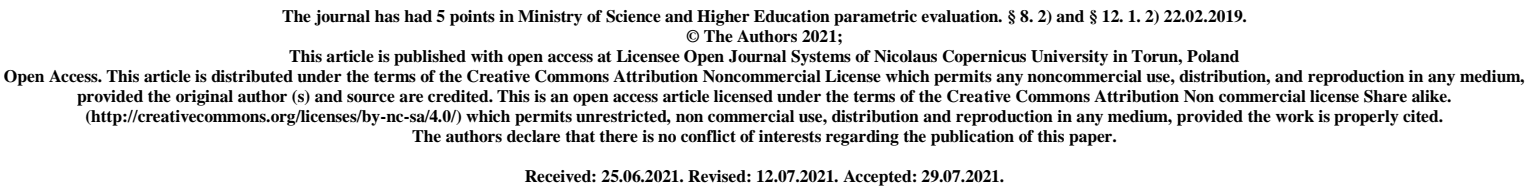

UDC 616.895.8-009.83

\title{
SOCIAL ADAPTIVE POTENTIAL OF MENTALLY ILL PATIENTS WITH CONCOMITANT PATHOLOGY
}

\author{
Ye. V. Oprya, E. V. Melnyk, Ya. V. Biesieda, Ye. V. Kozishkurt
}

\section{Odessa National Medical University, Odessa, Ukraine}

\begin{abstract}
The paper presents the results of research devoted to determination of social-adaptive potential of schizophrenic patients with concomitant somatic pathology by analyzing the parameters of socio-personal functioning, quality of life and compliance. The analysis of social and personal functioning of patients was carried out according to the results of the PSP scale (Personal and Social Performance Scale), which allowed to assess the social status of patients and their functioning in certain areas of life.

The quality of life of patients with schizophrenia in the surveyed groups was assessed using the results of the health questionnaire The 36-Item Short Form Survey (SF-36). According to the results of the research, it was found that when schizophrenia is combined with somatic disorders, there is a decrease in the level of socio-personal functioning, quality of life and compliance of patients. The social and personal functioning of patients with schizophrenia in its combination with somatic disorders was especially complicated in the areas of socially useful activities and self-care
\end{abstract}


(in schizophrenia with obesity) and in the field of social relations and restless/aggressive patterns of behavior (in schizophrenia with DM 2).

According to the assessment of quality of life in certain areas, it was found that in patients with schizophrenia with CVD and obesity, there was a decrease in physical functioning and limitation of daily functioning due to physical condition, as well as mental depression (the presence of severe depression and anxiety); in patients with schizophrenia with DM 2 - a decrease in daily functioning, due to emotional state; and in patients with obesity - also a decrease in social functioning; and a general decline in general health that was common to all patients with somatic disorders. The decrease in the level of compliance of patients with schizophrenia with somatic disorders was due to insufficient formation of factors related to the patient (perception and awareness of the disease, features and severity of symptoms, relapses, features of social functioning and adaptation) and attitudes towards treatment. According to the data obtained, the most dangerous in focus of social functioning it was the combination of schizophrenia with obesity.

The results of the study show that the combination of schizophrenia with somatic diseases is associated with a decrease in social functioning, quality of life and compliance with patients, which leads to a negative social prognosis and requires the attention of clinicians.

Key words: mental pathology; schizophrenia; concomitant somatic pathology; social adaptive potential; quality of life.

Introduction. It is noted that the presence of somatic diseases significantly complicates the conduct of adequate psychopharmacotherapy and significantly worsens the course and outcome of mental disease. Somatic pathology causes a 2-3 times increase in mortality from mental disorders and addictions and a decrease in life expectancy by 10-25 years [1].

According to modern research, mental disorders and addictions, and in particular schizophrenia and schizophrenic spectrum disorders, are associated with a high incidence of concomitant somatic diseases, the level of which is much higher than in the general population [2].

Recent studies have shown that the most common somatic diseases, which are combined with mental disorders and addictions, include cardiovascular pathology (including hypertension $20 \%$ ), type 2 diabetes (19\%), hyperlipidemia (14\%). and cancer [3]. 
Studies of the quality of life of patients are attracting increasing interest around the world $[4,5]$. Quality of life indicators include an assessment of the most important aspects of human life: social, mental and physical. This is especially important for patients with comorbid conditions (a combination of mental and somatic pathology, or different mental states), the coexistence of which significantly changes the clinical and psychopathological picture, sometimes masks the manifestation of a condition and requires other diagnostic methods and parameters of necessary medical care.

Studying the parameters of quality of life helps to determine the amount of medical care needed by the patient for faster readaptation, to assess the effectiveness of therapy, to optimize treatment, opens opportunities to influence on indicators such as motivation to treat and establish effective psychotherapeutic contact, so-called compliance. Taking into account the indicators of quality of life, it is possible to make a targeted and balanced medical and psychosocial impact.

At the present stage, this indicator is considered, along with clinical data, as a diagnostic criterion. Many researchers emphasize that quality of life indicators are new objective criteria for providing medical care $[6,7]$. These indicators are used for planning and operation of medical services, in the study of clinical features of the disease and treatment results [8]. Undoubtedly, this directly refers to diseases that are combined and require different medical tactics.

The level of social functioning is recognized as one of the key criteria for assessing the patient's condition, and his improvement is an objective criterion for the effectiveness of treatment.

Aim of the work: In this study, the social-adaptive potential of patients with schizophrenia was determined by analyzing the parameters of socio-personal functioning, quality of life and compliance.

\section{Material and methods.}

In accordance with the purpose and objectives of the study, three main and one control group of the study were formed. The main research groups consisted of 136 patients with schizophrenia, which was combined with somatic diseases. In total, three main groups were formed.

The first main group of the study consisted of 50 patients with schizophrenia with chronic cardio-vascular disease (CVD), which at the time of the study were compensated. Patients with post-stroke and post-infarction conditions were not included in the study. 
The second main group of the study presented 42 patients with schizophrenia with type 2 diabetes mellitus (DM 2) without signs of obesity (BMI<30).

The third main group of the study consisted of 44 patients with schizophrenia with a diagnosis of obesity (provided that the body mass index (BMI $\geq 30)$.

The control group was formed from patients with schizophrenia without chronic somatic disorders and numbered 50 people.

The following methods were used as research tools: psychometric, psychodiagnostic and clinical-statistical. The analysis of social and personal functioning of patients was carried out according to the results of the PSP scale (Personal and Social Performance Scale), which allowed to assess the social status of patients and their functioning in certain areas of life. The quality of life of patients with schizophrenia in the surveyed groups was assessed using the results of the health questionnaire The 36-Item Short Form Survey (SF-36).

Results. According to the results of the research, it was found that when schizophrenia is combined with somatic disorders, there is a decrease in the level of socio-personal functioning, quality of life and compliance of patients.

Patients with schizophrenia of all examined groups were characterized by the presence of significant complications in socio-personal functioning, with the lowest level of social and personal functioning was observed in patients with schizophrenia with obesity $(43.4 \pm 1.72$ points), compared with the control group $(54,7 \pm 1.88$ points $)$, at $\mathrm{p} \leq 0.05$. The level of social and personal functioning of patients with schizophrenia with cardiovascular disease (CVD) was (50.5 \pm 2.11 points), patients with schizophrenia with diabetes mellitus type 2 (DM 2) (48.2 \pm 1.93 points).

The social and personal functioning of patients with schizophrenia in its combination with somatic disorders was especially complicated in the areas of socially useful activities and selfcare (in schizophrenia with obesity) and in the field of social relations and restless/aggressive patterns of behavior (in schizophrenia with DM 2).

It was determined that patients with schizophrenia with somatic disorders rated the overall quality of life lower, especially patients with CVD (49.61\% points) and obesity (48.54\% points), compared with the control group (59.05\% points), at $\mathrm{p} \leq 0.05$. According to the assessment of quality of life in certain areas, it was found that in patients with schizophrenia with CVD and obesity, there was a decrease in physical functioning and limitation of daily functioning due to 
physical condition, as well as mental depression (the presence of severe depression and anxiety); in patients with schizophrenia with DM 2 - a decrease in daily functioning, due to emotional state; and in patients with obesity - also a decrease in social functioning; and a general decline in general health that was common to all patients with somatic disorders.

The level of compliance of patients with schizophrenia with somatic disorders was lower than in the control group, it was especially low in patients with obesity (56.14\%), compared with the control group (66.04\%), at $\mathrm{p} \leq 0.05$. The level of compliance of patients with schizophrenia with DM 2 was $58.04 \%$, in patients with schizophrenia with CVD - 62.48\%, but without a significant difference with the control group. The decrease in the level of compliance of patients with schizophrenia with somatic disorders was due to insufficient formation of factors related to the patient (perception and awareness of the disease, features and severity of symptoms, relapses, features of social functioning and adaptation) and attitudes towards treatment (attitude towards drugs, fears and anxieties about side effects, satisfaction with the results of therapy, etc.).

According to the data obtained, the most dangerous in focus of social functioning it was the combination of schizophrenia with obesity.

The results of the study show that the combination of schizophrenia with somatic diseases is associated with a decrease in social functioning, quality of life and compliance with patients, which leads to a negative social prognosis and requires the attention of clinicians.

\section{References}

1. Prevalence of Combined Somatic and Mental Health Multimorbidity: Patterns by Age, Sex, and Race / Ethnicity / Bobo W.V., Yawn B.P., Sauver J.L. St. [et al.] // The Journals of Gerontology Series A: Biological Sciences and Medical Sciences. - 2016. -Vol. 11 (71). - P. 1483-1491.

2. Comorbidities with chronic physical conditions and gender profiles of illness in schizophrenia. Results from PREST, a new health dataset // A. Gabilondo, E. Alonso-Moran, R. Nuño-Solinis [et al.] // J Psychosom Res. - 2017. - Vol. 93. - P.102-109.

3. Somatic Comorbidity in Schizophrenia: Some Possible Biological Mechanisms Across the Life Span / I. Dieset [et al.] // Schizophrenia Bulletin. - 2016. - Vol. 42, Issue 6. - P. 1316-1319. 
4. Colombo F. Addressing multimorbidity to improve healthcare and economic sustainability / F. Colombo, M. García-Goñi, C. Schwierz // Journal of Comorbidity. - 2016. Vol. 6 (1). - P. 21-27.

5. Cohen A. Addressing comorbidity problems between mental disorders and major noncommunicable diseases. Background technical paper to support the implementation of the European Mental Health Action Plan 2013-2020 and the WHO Action Plan for the Prevention and Control of Noncommunicable Diseases in the WHO European Region 2016-2025. World health organization. - 2017. - 44 p. [In Russian].

6. Overview of the European Framework for Action on Integrated Health Services Delivery. Copenhagen: WHO Regional Office for Europe. - 2016. - 21 p.

7. Belyalov F.I. Treatment of diseases in conditions of comorbidity. M. : GEOTARMedia; 2016. - 264 p. [In Russian].

8. Epidemiology and Psychiatric Sciences / J. Westman, S.V.Eriksson, M. Gissler [et al.]. - 2018. - Vol. 27, Issue 5. - P. 519-527. 